ORIGINAL ARTICLE

\title{
Pilot study for the development of a new campylobacter selective medium at $37^{\circ} \mathrm{C}$ using aztreonam
}

\author{
G D Thomas
}

J Clin Pathol 2005;58:413-416. doi: 10.1136/icp.2004.019042

\begin{abstract}
Aims: To overcome contamination and temperature inhibition by isolating campylobacter at $37^{\circ} \mathrm{C}$.
Methods: The $\beta$ lactam antibiotic aztreonam was included in a selective medium because of its inhibitory activity against Gram negative organisms but not against Campylobacter jejuni. Vancomycin and amphotericin were added to inhibit Gram positive bacteria and yeasts.

Results: The aztreonam amphotericin vancomycin (AAV) experimental campylobacter selective medium showed growth microaerobically at $37^{\circ} \mathrm{C}$ of $\mathrm{C}$ jejuni, $\mathrm{C}$ coli, $\mathrm{C}$ lari, $\mathrm{C}$ hyointestinalis, $C$ fetus subsp. fetus, and $C$ jejuni subsp. doylei after 24 to 48 hours of incubation. Six campylobacter NCTC strains demonstrated a minimum inhibitory concentration $(\mathrm{MIC}) \geqslant 256 \mathrm{mg} /$ litre for vancomycin and aztreonam, whereas C upsaliensis and two "campylobacter-like" strains now reclassified under genus helicobacter$H$ cinaedi and $H$ fennelliae-had a MIC of $4 \mathrm{mg}$ /litre for vancomycin and aztreonam. In the pilot study (150 samples), AAV medium $\left(37^{\circ} \mathrm{C}\right)$ had a higher sensitivity for isolating campylobacters: 14 were isolated on AAV compared with 10 on modified $\operatorname{CDA}\left(43^{\circ} \mathrm{C}\right)$ over three days, and nine were isolated on AAV medium compared with five on modified $\mathrm{CDA}\left(43^{\circ} \mathrm{C}\right)$ after 24 hours of incubation. Contamination rates remained low.

Conclusion: The medium was devised in a pilot study performed between 1990 and 1993; however, this is

the first report of AAV medium used as a selective medium capable of growing six campylobacters of pathogenic importance at $37^{\circ} \mathrm{C}$. Further studies are indicated.
\end{abstract}

Correspondence to:

G D Thomas,

lpi@lpi37.com

Accepted for publication

15 October 2004

\begin{abstract}
C ampylobacter isolation requires a simple medium that uses antibiotics as selective agents. These antibiotics vary from the "cocktail" of vancomycin, polymixin B, and trimethroprin found in Skirrow's medium, ${ }^{1}$ to a single antibiotic (cefazolin) in $\mathrm{CDA}^{2}$ and cefoperazone in modified $\mathrm{CDA}^{3}$; in addition, less inhibitory agents have also been advocated, such as teicoplanin in CAT medium. ${ }^{4}$ However, Campylobacter jejuni and $C$ coli remain the two main pathogens responsible for campylobacter enteritis, and early diagnosis by culture and isolation can enhance clinical investigation. The recovery of campylobacters remains dependant on the selective media and techniques used, and in their letter to the editor, Bolton et al raised the question: "what are we missing?". ${ }^{5}$ A large number of comparative studies and evaluations of conventional methods have preceded and followed that question, ${ }^{6-8}$ with McClurg and colleagues ${ }^{9}$ repeating the question 15 years later; fundamentally, studies have described the temperature of $43^{\circ} \mathrm{C}$ as prohibitive for the growth of some $C$ lari species, ${ }^{6}$ and the selective pressure of the antibiotics cefalothin, colistin, and polymixin $\mathrm{B}$, normally incorporated into most conventional media, as a reason for the low recovery of some strains of $C$ jejuni, $C$ coli, $C$ hyointestinalis, and non-thermophiles. ${ }^{10}{ }^{11}$ More recent studies have found that filtration techniques are effective but time consuming, ${ }^{4}$ and that molecular methods of detecting campylobacters using DNA probes are not ideal for routine clinical use. ${ }^{12}$
\end{abstract}

"Studies have described the temperature of $43^{\circ} \mathrm{C}$ as prohibitive for the growth of some Campylobacter lari subspecies"

* NCTC strain C jejuni subsp. doylei became contaminated during the study
A preliminary study evaluating conventional methods ${ }^{6}$ was inconclusive, so this present pilot study sought to establish an isolation selective medium that would support the growth of most common enteric campylobacters. The study evaluates six different antibiotics not previously used in campylobacter selective medium, with the hope that one antibiotic would prove active in microaerobic conditions, suppress Gram negative flora, and yet be non-inhibitory to Campylobacter spp at the incubation temperature of $37^{\circ} \mathrm{C}$. Aztreonam promised to fulfilled these requirements. Furthermore, vancomycin was chosen because of its inhibitory effect on Gram positive contaminating flora, and was assessed for its antagonism to aztreonam; amphotericin was also included in the selective combination for its activity against yeasts. This pilot study for AAV medium used several challenge methods and AAV was compared with modified CDA medium to verify its subsequent suitability as a selective medium for the isolation of Campylobacter spp.

\section{METHODS AND MATERIALS}

\section{Selective media, cultures, and incubation}

Modified CDA: colombia blood agar (Oxoid CM 351; Oxoid, Basingstoke, UK) plus 7\% (wt/vol) defibrinated horse blood. $\mathrm{AAV}$ was developed as follows: campylobacter charcoal base (Oxoid CM 908), $10 \mathrm{mg} /$ litre aztreonam (Squib, Moreton, UK), dissolved in $\mathrm{H}_{2} \mathrm{O}$ (refrigerated for 24 hours before use), $2 \mathrm{mg} /$ litre amphotericin (Squib), and $10 \mathrm{mg} /$ litre vancomycin (hydrochloride) (Lilly, Basingstoke, UK). NCTC strains of campylobacter obtained from NCTC were as follows: $C$ jejuni NCTC 11168, C coli NCTC 11366, C lari NCTC 11352, C hyointestinalis, C jejuni subsp. doylei* ${ }^{*}$ C fetus subsp. fetus, and C upsaliensis NCTC 11926. In addition, two "campylobacterlike" strains now reclassified under genus helicobacter were

Abbreviations: AAV, aztreonam amphotericin vancomycin; MIC, minimum inhibitory concentration 
Table 1 Suitability study showing susceptibility of Campylobacter spp to the six antibiotics tested

\begin{tabular}{|c|c|c|c|c|c|}
\hline \multirow[b]{2}{*}{ Antibiotic disc } & \multicolumn{5}{|c|}{ Campylobacter strains } \\
\hline & C fetus & C jejuni & C hyointestinalis & C coli & C lari \\
\hline Imipenem (10 mg) & S & S & $S$ & $\mathrm{~S}$ & S \\
\hline Amikacin (10 mg) & $\mathrm{S}$ & $\mathrm{S}$ & S & S & S \\
\hline Tobramycin (10 mg) & $S$ & $\mathrm{~S}$ & S & S & $S$ \\
\hline Azlocillin $(75 \mathrm{mg})$ & $\mathrm{S}$ & $\mathrm{S}$ & S & S & S \\
\hline Piperacillin (75 mg) & $\mathrm{S}$ & S & S & S & $\mathrm{R}$ \\
\hline \multirow[t]{2}{*}{ Aztreonam (30 mg) } & $\mathrm{R}$ & $\mathrm{R}$ & $\mathrm{R}$ & $\mathrm{R}$ & $\mathrm{R}$ \\
\hline & C upsaliensis & $H$ cinaedi & H fennelliae & & \\
\hline Aztreonam (30 mg) & S & S & S & & \\
\hline
\end{tabular}

tested: H fennelliae NCTC 11612 and H cinaedi NCTC 11611. A reservoir of campylobacter strains and routine isolates from a preliminary study was preserved for suitability studies. Microaerobic incubation, identification, and biotyping procedures were adapted from Bolton et al, ${ }^{6}$ and efficacy, minimum inhibitory concentration (MIC), and challenge procedures by multipoint inoculation were adapted from Karmali et al. ${ }^{7}$

Suitability studies: untried antibiotics and antagonism The suitability of the untried antibiotics was assessed by a disc diffusion method, in which six commercially prepared discs (Mast, Bootle, UK) chosen for their inhibitory action against Gram negative organisms were placed on to each lawned control strain. The discs contained the following antibiotics: tobramycin $(10 \mathrm{mg})$, azlocillin $(75 \mathrm{mg})$, imipenem $(10 \mathrm{mg})$, amikacin $(10 \mathrm{mg})$, piperacillin $(75 \mathrm{mg})$, and aztreonam $(30 \mathrm{mg})$, and zones of inhibition were recorded. Any possible antagonism when in combination with vancomycin was established by placing the commercially prepared discs of aztreonam (30 mg) and vancomycin $(30 \mathrm{mg}) 15 \mathrm{~mm}$ apart on non-selective blood plates, lawned with Oxford staphylococcus NCTC 6571 and Escherichia coli NCTC 10418 as controls, and inhibition zones were noted for radial symmetry.

\section{Evaluation of prototype AAV medium}

The suitability of the selective prototype medium was assessed by comparing it with modified CDA; 454 routine isolates-of which 385 were coliforms and 69 Gram positives-were spot inoculated in $0.3 \mu \mathrm{l}$ volumes by a multipoint inoculation technique (Mast) on to modified CDA and AAV medium, growth selected, and saved before identification and MIC determination of aztreonam and vancomycin.

One hundred and fifty hospital faecal samples were routinely cultured and all plates incubated microaerobically for three days with AAV medium at $37^{\circ} \mathrm{C}$ and modified CDA at $43^{\circ} \mathrm{C}$. Procedures were standardised with $C$ jejuni NCTC 11168 and plates read on successive days for three days. Suspect campylobacter growth was confirmed by oxidase, Gram film, motility, and characteristic morphology, with isolates on AAV medium saved for identification and MIC determination.

\begin{tabular}{|c|c|c|c|c|}
\hline & Day 1 & Day 2 & Day 3 & \\
\hline Medium & $\begin{array}{l}\text { No of } \\
\text { isolates }\end{array}$ & $\begin{array}{l}\text { No of } \\
\text { isolates }\end{array}$ & $\begin{array}{l}\text { No of } \\
\text { isolates }\end{array}$ & Total (\%) \\
\hline $\begin{array}{l}\text { Modified } \\
\text { CDA }\end{array}$ & 5 & 4 & 1 & $10(7 \%)$ \\
\hline AAV & 9 & 4 & 1 & $14(9 \%)$ \\
\hline
\end{tabular}

\section{Evaluation of prototype: growth efficacy, cephalothin, and thermotolerance}

Methods used a comparison of AAV medium with modified CDA, with serial 10 fold dilutions of NCTC campylobacter strains to $10^{-10}(\mathrm{vol} / \mathrm{vol})$ spot inoculated by the multipoint method on to modified CDA and AAV media. All plates were incubated microaerophilically at $37^{\circ} \mathrm{C}$ for 24 hours, and results were recorded as the lowest dilution to show visible growth. Campylobacters not isolated by modified CDA, but which grew on AAV at $37^{\circ} \mathrm{C}$, were re-challenged for susceptibility (inoculum diluted to $10^{-10}$ by plate-out) by plating on to non-selective charcoal agar at $37^{\circ} \mathrm{C}$ at $43^{\circ} \mathrm{C}$ using a cefradine disc of $30 \mathrm{mg}$, and incubated under microaerobic conditions for 48 hours.

\section{MIC, biotyping, and identification}

Doubling dilutions of the antibiotics vancomycin and aztreonam were prepared in a charcoal agar base in the range $0.2-256 \mathrm{mg} / \mathrm{litre}$, and preserved NCTC campylobacter strains, clinical strains, and routine contaminants that grew on AAV medium $\left(37^{\circ} \mathrm{C}\right)$ were recultured and spot inoculated on to each set of prepared plates. Growth was recorded by Mastascan and MIC values read as the lowest concentration giving visible growth. The coliforms were identified using the API E kit (bioMérieux, Marcy-L'Etoile, France), and a simplified biotyping scheme adapted from Penner ${ }^{13}$ was used for the identification of campylobacters.

Table 2 Challenge studies showing growth of routine isolates (using multipoint) tested against modified $C D A\left(43^{\circ} \mathrm{C}\right)$ and $A A V$ $\left(37^{\circ} \mathrm{C}\right)$ medium

\begin{tabular}{|c|c|c|c|c|c|c|}
\hline Medium & $\begin{array}{l}\text { Total no of } \\
\text { organisms tested }\end{array}$ & $\begin{array}{l}\text { Total no of } \\
\text { coliforms }\end{array}$ & $\begin{array}{l}\text { Total no of Gram } \\
\text { positives }\end{array}$ & $\begin{array}{l}\text { Coliforms resistant } \\
\text { on multipoint set }\end{array}$ & $\begin{array}{l}\text { Gram positives resistant on } \\
\text { multipoint set }\end{array}$ & $\begin{array}{l}\text { Total resistant } \\
\text { organisms }\end{array}$ \\
\hline Modified CDA & 454 & 385 & 69 & $16(3 \%)$ & $2(0.5 \%)$ & $18(4 \%)$ \\
\hline AAV & 454 & 385 & 69 & $22(5 \%)$ & 0 & $22(5 \%)$ \\
\hline
\end{tabular}

AAV, aztreonam amphotericin vancomycin. 
Table 4 Numbers of plates contaminated over three days of incubation on modified $\operatorname{CDA}\left(43^{\circ} \mathrm{C}\right)$ and $\mathrm{AAV}$ medium $\left(37^{\circ} \mathrm{C}\right)$ (150 stool samples)

\begin{tabular}{|c|c|c|c|c|c|c|}
\hline & \multicolumn{2}{|l|}{ Day 1} & \multicolumn{2}{|l|}{ Day 2} & \multicolumn{2}{|l|}{ Day 3} \\
\hline & $\begin{array}{l}\text { Modified } \\
\text { CDA }\end{array}$ & AAV & $\begin{array}{l}\text { Modified } \\
\text { CDA }\end{array}$ & AAV & $\begin{array}{l}\text { Modified } \\
\text { CDA }\end{array}$ & AAV \\
\hline \pm & - & - & 1 & 7 & 2 & 4 \\
\hline+ & 4 & 5 & 6 & 9 & 3 & 8 \\
\hline++ & 10 & 3 & 2 & 2 & 4 & - \\
\hline +++ & 7 & - & 1 & - & - & 3 \\
\hline TL & - & - & - & 10 & 1 & 6 \\
\hline Total & 21 (14\%) & $8(5 \%)$ & $10(7 \%)$ & $28(19 \%)$ & $10(7 \%)$ & 21 (14\%) \\
\hline
\end{tabular}

$\mathrm{AAV}$, aztreonam amphotericin vancomycin; $\mathrm{TL}$, thin layer; \pm , very scanty growth; +, scanty growth; ++ , moderate growth; +++ , heavy growth.

\section{RESULTS}

\section{Suitability studies}

The aztreonam disc $(30 \mathrm{mg})$ showed no inhibitory action against $C$ jejuni NCTC 11168, $C$ coli NCTC 11366, $C$ lari NCTC 11352, C jejuni subsp. doylei, C hyointestinalis, C fetus subsp. fetus (table 1), although it did not inhibit $C$ upsaliensis NCTC 11926, $H$ fennelliae NCTC 11612, and H cinaedi NCTC 11611. A combination of aztreonam and vancomycin showed no antagonism and supported the growth of all campylobacter NCTC strains with the exception of $C$ upsaliensis NCTC 11926, $H$ fennelliae NCTC 11612, and H cinaedi NCTC 11611 ; however a "wild-type" clinical strain of $C$ upsaliensis grew on AAV medium after 48 hours of incubation (table 1).

\section{Challenge studies: isolation and contamination}

No significant difference was shown between the selectivity of both media against Gram negative bacteria: 22 coliforms grew on AAV medium compared with 16 on modified CDA; however, the growth of all Gram positive bacteria tested was inhibited by AAV medium (table 2 ). From 150 routine patient faecal samples, 14 (9\%) campylobacters were isolated on AAV medium at $37^{\circ} \mathrm{C}$, nine of which grew after 24 hours of incubation, compared with $10(7 \%)$ campylobacters on modified CDA $\left(43^{\circ} \mathrm{C}\right)$, five of which grew after 24 hours of incubation (table 3). After one day of incubation, contamination on AAV medium $\left(37^{\circ} \mathrm{C}\right)$ remained minimal, with $5 \%$ of plates demonstrating mainly coliform contaminants-for example, E coli, Enterobacter cloacae, Citrobacter freundi, and a rare colony of Acinetobacter spp, compared with 14\% contamination on modified $\mathrm{CDA}\left(43^{\circ} \mathrm{C}\right)$. If contamination was assessed separately for each day, a reverse trend was seen on the second and third days when $19 \%$ and $14 \%$ of AAV plates $\left(37^{\circ} \mathrm{C}\right)$, respectively, showed contamination by facultative aerobic organisms (such as Lactobacilli spp, Enterococcus spp, and yeasts) compared with $7 \%$ of modified CDA plates $\left(43^{\circ} \mathrm{C}\right)$ on both days (table 4$)$.

\section{Growth efficacy, biotyping, and MIC values}

AAV medium proved slightly more successful in supporting the growth of campylobacter NCTC strains, but $C$ upsaliensis NCTC 11926, H fennelliae NCTC 11612, and H cinaedi NCTC 11611 did not grow on this medium. Of the 14 campylobacters isolated, 10 were identified as $C$ jejuni; two as $C$ coli, one of which was isolated on both $\operatorname{AAV}\left(37^{\circ} \mathrm{C}\right)$ and modified CDA $\left(43^{\circ} \mathrm{C}\right)$ on the third day of incubation (table 3 ), and the second of which was isolated only on $\operatorname{AAV}\left(37^{\circ} \mathrm{C}\right)$; the two remaining campylobacters that grew were identified as C jejuni subsp. doylei. All campylobacter NCTC strains showed a MIC $\geqslant 256 \mathrm{mg} /$ litre to aztreonam and vancomycin, with the exception of $C$ upsaliensis NCTC 11926, H fennelliae NCTC 11612 , and $H$ cinaedi NCTC 11611 , each with a MIC of $4 \mathrm{mg} /$ litre to aztreonam and vancomycin (table 5). The MIC values for campylobacter strains from earlier work and the pilot study were within the range of 128-256 mg/litre, with one exception being provided by a $C$ jejuni isolate from a child, which had a MIC value of $128 \mathrm{mg} /$ litre. The eight coliforms that failed to grow when re-challenged on AAV medium gave MIC values of 2-128 mg/litre for aztreonam and a MIC value $\geqslant 256 \mathrm{mg} /$ litre for vancomycin; in addition, the four Lactobacilli spp, with MIC values of 32-64 mg 1/litre for vancomycin and MIC values of 128-256 mg/litre for aztreonam, were sensitive when re-challenged. An Enterococcus spp re-grew on AAV medium giving a MIC value of $\geqslant 256 \mathrm{mg} /$ litre for both aztreonam and vancomycin.

\section{DISCUSSION}

No one medium fulfils all the criteria for the isolation of campylobacter; this phrase remains the topic for a discussion for campylobacter isolation. In this study, aztreonam proved to be non-inhibitory for six campylobacter NCTC reference strains and, importantly, vancomycin was found to be nonantagonistic to aztreonam. Therefore, it was initially thought that campylobacters might be inherently resistant to aztreonam, because antibiotic susceptibility studies by Rittenbury ${ }^{14}$ had shown that the $\beta$ lactam antibiotic aztreonam bound poorly to the penicillin binding protein sites of microaerophilic and aerobic bacteria. However, this theory was quickly disproved with the sensitivity of $C$ upsaliensis NCTC 11926 to aztreonam. AAV medium supported the growth of six NCTC campylobacter reference strains and grew a "wild-type" clinical strain of $C$ upsaliensis, and some complimentary antibiotic activity was exhibited between the antibiotics aztreonam and vancomycin. This last fact would enhance selective pressure and provide an explanation for the equal activity of AAV medium to modified CDA in inhibiting the growth of coliforms and Gram positive contaminants.

In a small comparative study ( 150 samples), $\operatorname{AAV}\left(37^{\circ} \mathrm{C}\right)$ was more successful in isolating campylobacters on the first day of incubation: nine campylobacters were isolated on AAV $\left(37^{\circ} \mathrm{C}\right)$ medium compared with five on modified CDA $\left(43^{\circ} \mathrm{C}\right)$. Medium efficacy provides one explanation in that $\mathrm{AAV}$ medium showed more ability to support the growth of

Table 5 Identification and minimum inhibitory concentration (MIC) values of campylobacter reference strains

\begin{tabular}{|c|c|c|c|}
\hline Campylobacter reference strains & Identification & MIC vancomycin & MIC aztreonam \\
\hline $\begin{array}{l}\text { C jejuni* NCTC } 11168 \\
\text { C lari NCTC } 11352 \\
\text { C coli NCTC } 11366 \\
\text { C fetus subsp. fetus } \\
\text { H (C) fennelliae NCTC } 11612 \\
\text { H (C) cinaedi NCTC } 11611 \\
\text { C upsaliensis NCTC } 11926 \\
\text { C hyointestinalis }\end{array}$ & $\begin{array}{l}\text { C jejuni } \\
\text { C lari } \\
\text { C coli } \\
\text { C fetus } \\
H(C) \text { fennelliae } \\
H(C) \text { cinaedi } \\
\text { C upsaliensis } \\
\text { C hyointestinalis }\end{array}$ & $\begin{array}{l}>256 \\
>256 \\
>256 \\
>256 \\
>256 \\
>256 \\
>256 \\
>256\end{array}$ & $\begin{array}{l}>256 \\
>256 \\
>256 \\
>256 \\
4 \\
4 \\
4 \\
>256\end{array}$ \\
\hline
\end{tabular}




\section{Take home messages}

- AAV (aztreonam amphotericin vancomycin) campylobacter selective medium supported the growth of six campylobacter strains, although it did not support the growth of the C upsaliensis NCTC strain

- In the pilot study (150 samples), AAV medium improved rates of isolation, particularly on the first day, which could be clinically relevant

- Contamination rates were low

- Further studies are indicated

Campylobacter spp, but temperature and cephalothin tolerance studies provided no clear indication of why four more campylobacters were isolated on AAV medium at $37^{\circ} \mathrm{C}$. All four strains grew equally well at $37{ }^{\circ} \mathrm{C}$ and $43^{\circ} \mathrm{C}$ on nonselective charcoal medium and all were resistant to cephalothin. Equally, each isolate gave MIC values $\geqslant 256 \mathrm{mg} /$ litre for vancomycin and aztreonam, but one isolate was identified as $C$ coli, which is generally more fastidious than $C$ jejuni. It would have been useful to have estimated MIC values for cefoperazone, but time limits did not permit this. AAV medium $\left(37^{\circ} \mathrm{C}\right)$ showed good selective pressure to faecal flora, with $5 \%$ of plates contaminated after the first day of incubation compared with $14 \%$ on modified CDA $\left(43^{\circ} \mathrm{C}\right)$; however, a change in the preparation of the AAV plates (the addition of $1 \mathrm{ml}$ of 7\% horse blood) affected 30-40 plates and coincided with an increase number of contaminants growing on the second and third day of incubation. This reverse trend was temporary, and contaminants were identified as resistant Lactobacilli spp and diphthroids, which had MIC values $>256 \mathrm{mg} /$ litre for aztreonam and vancomycin. Because these MIC values differed from the other Lactobacilli spp isolated in the study, these organisms may have derived from the reagents and not from the faeces. However, it was shown that blood protein probably bound to the deoxycholate component of the charcoal basal agar, reducing the inhibitory effect of deoxycholate over contaminants resistant to aztreonam and vancomycin. Once this procedure was discontinued, and 7\% horse blood omitted from the preparation of the AAV plates, rates of contamination returned to the same proportions seen on the first day of incubation; however, the overall results were slightly distorted because of this additional procedure.

"This early confirmation of culture and isolation could remove some of the delays seen at present and provide the clinician with an option for rapid treatment"

Of the eight coliform contaminants sensitive when rechallenged on AAV, records showed that one $E$ coli with a MIC of $128 \mathrm{mg} /$ litre was derived from a patient receiving multiple antibiotic treatment; thus, acquired antibiotic resistance $^{15}$ is one possible explanation for the initial growth on AAV medium. With the exception of one $C$ jejuni isolate with a MIC value of $128 \mathrm{mg} /$ litre for aztreonam, campylobacter NCTC strains, reserve strains, and those isolated from the study (50 strains) all had MIC values $\geqslant 256 \mathrm{mg} /$ litre for vancomycin and aztreonam. The C upsaliensis NCTC 11926 with a MIC value of $4 \mathrm{mg} /$ /itre was the only campylobacter sensitive to aztreonam, and genetic modification ${ }^{15}$ may provide an explanation of why there was growth of the wild-type clinical strain in the suitability studies.

AAV medium at $37^{\circ} \mathrm{C}$ improved rates of isolation on the first day of incubation; this early confirmation of culture and isolation could remove some of the delays seen at present and provide the clinician with an option for rapid treatment. Use of the medium enabled the easy recognition of campylobacter growth, with the culture presenting as a silver grey swarming colony, and facilitated the isolation of C coli, C fetus subsp. fetus, $C$ lari, C hyointestinalis, and C jejuni subsp. doylei without the conventional methods of filtration or the need of incubation at $43^{\circ} \mathrm{C}$. The pilot study was small (150 samples) and aztreonam was slightly denatured by acidity under in vitro conditions; the buffering of the medium at $37^{\circ} \mathrm{C}$, the type of sample, or the clinical history of the patient may be the responsible factor. Therefore, further studies are needed.

G D Thomas, previously of Sheffield Children's Hospital (UK)

\section{REFERENCES}

1 Skirrow MB. Campylobacter enteritis. A new disease. BMJ 1977;25:10-12.

2 Bolton FJ, Hutchinson DN, Coates G. Blood-free selective medium for isolation of Campylobacter jejuni from faeces. J Clin Microbiol 1984;19:169-71.

3 Hutchinson DN, Bolton FJ. An improved blood free selective medium for the isolation of Campylobacter jejuni from faecal specimens. J Clin Pathol 1984;37:956-7.

4 Aspinall ST, Waring DR, Hayward PG, et al. A comparison of a new selective medium (CAT) with membrane filter for the isolation of thermophilic campylobacter including C. upsaliensis. J Appl Bacteriol 1996;80:645-50.

5 Bolton FJ, Hutchinson DN, Parker G. Isolation of campylobacter; what are we missing? J Clin Pathol 1987;40:702-3.

6 Bolton FJ, Hutchinson DN, Parker G. Reassessment of selective agars and filtration technique for isolation of campylobacter species from faeces. Eur J Clin Microbiol Infect Dis 1988;7:155-60.

7 Karmali MA, Simor AE, Roscoe M, et al. Evaluation of a blood-free, charcoalbased selective medium for the isolation of campylobacter organisms from faeces. J Clin Microbiol 1986;23:456-59.

8 Engberg J, On SLW, Harrington CS, et al. Prevalence of Campylobacter, Archobacter, Helicobacter and Sutterella spp. in human faecal samples as estimated by a re-evaluation of isolation methods for campylobacters. J Clin Microbiol 2000;38:286-91.

9 McClurg KR, McClurg RB, Moore JE. Efficient isolation of campylobacters from stools: what are we missing? J Clin Pathol 2002;55:239-40.

10 Corry JE, Post DE, Colin P, et al. Culture media for the isolation of campylobacters. Int J Food Microbiol 1995;26:43-76.

11 Fennell CL, Totton PR, Quinn TC, et al. Characterisation of campylobacter-like organisms from homosexual men. J Infect Dis 1984;149:58-66.

12 Maher M, Finnigan C, Collins E, et al. Evaluation of culture methods and a DNA probe-based PCR assay for detection of campylobacter species in clinical specimens of faeces. J Clin Microbiol 2003;41:2980-6.

13 Penner JL. The genus campylobacter: a decade of progress. Clin Microbiol Rev 1988; 1:157-72.

14 Rittenbury MS. "How and why aztreonam works." Surg Gynecol Obstet Supp 1990;171:19-23.

15 Lau SKP, Woo PCY, Leung KW, et al. Emergence of cotrimoxazole and quinolone resistant campylobacter infections in bone marrow transplant recipient. Eur J Clin Infect Dis 2002;21:286-91. 